\title{
Parafascicular Nucleus of the Thalamus
}

National Cancer Institute

\section{Source}

National Cancer Institute. Parafascicular Nucleus of the Thalamus. NCI Thesaurus. Code C33260.

Part of the posterior (caudal) intralaminar group of thalamic nuclei, located medial to the centromedian nucleus and ventral to the dorsomedial nuclei, that is associated with the limbic and associative striatum. 